\title{
Certificación Global Ambiental: ¿Buenas intenciones?
}

\section{María Fernanda Silva Tejada}

En el presente artículo, la autora analizará la viabilidad de la Certificación Global Ambiental y también se identificarán los puntos críticos que el Estado debe fortalecer para que se cumplan los objetivos de la CGA.

Estudiante de la Facultad de Derecho de la Universidad del Pacífico. Artículo redactado el 3 de noviembre de 2015, ganador del XIV Taller de Derecho Ambiental de la Sociedad Peruana de Derecho Ambiental.

Esta publicación no habría sido posible sin los intercambios de ideas y discusiones respecto al tema de la Certificación Ambiental Global con los especialistas en el tema. Específicamente agradezco a Isabel Calle, directora del Programa de Política y Gestión Ambiental de la Sociedad Peruana de Derecho Ambiental (SPDA) y a Milagros Verástegui, asesora de la Alta Dirección del Servicio Nacional de Certificación Ambiental para las Inversiones Sostenibles (SENACE). 


\section{Certificación Global Ambiental: ¿Buenas intenciones?}

\section{Introducción}

Durante el gobierno de Ollanta Humala, se agudiza el cuestionamiento de la legitimidad de los Estudios de Impacto Ambiental (EIA). La pérdida de confianza en este instrumento se evidenció en el año 2011 con el conflicto del proyecto Conga en Cajamarca ${ }^{1}$, donde incluso el respectivo EIA fue sometido a un arbitraje internacional. No obstante ello, el proyecto no fue aceptado y a su vez generó la caída de dos gabinetes ministeriales en el primer año de esta gestión gubernamental ${ }^{2}$.

Por otro lado, el Reporte Mensual de Conflictos Sociales de la Defensoría del Pueblo destaca que el mayor componente de la conflictividad en el Perú son los enfrentamientos de tipo socio-ambiental asociados a las principales actividades extractivas que requieren de EIA para su operación, los cuales para el período 2012 - setiembre 2015 superan el $65 \%$, a comparación al registrado en diciembre del 2011 , que fue de $56.5 \%{ }^{3}$. Estos conflictos se presentan, fundamentalmente, en la actividad minera, la cual tiene un aporte importante en el crecimiento económico de nuestro país ${ }^{4}$.

En este contexto, ante la agudización de los conflictos socio-ambientales de actividades extractivas, se crea el Servicio Nacional de Certificación Ambiental para las Inversiones Sostenibles (SENACE) ${ }^{5}$. No obstante esta innovación en la gestión ambiental, el Perú ha perdido competitividad a nivel mundial de acuerdo al Informe Global de Competitividad del Foro Económico Mundial para el período 2014-2015.

1 De Echave, José y Diez, Alejandro, "Más allá de Conga", Red Peruana por una Globalización con Equidad - RedGE, marzo 2013.

2 De Echave, José y Diez, Alejandro, "Más allá de Conga", Red Peruana por una Globalización con Equidad - RedGE, marzo 2013.

3 Porcentajes tomados de los Reportes Mensuales de Conflictos Sociales de la Defensoría del Pueblo estimados al mes de diciembre de cada año. Disponible en: http://www.defensoria.gob.pe/conflictos-sociales/home.php?sec=1\&pag=2.

Última consulta 30 de octubre del 2016. Defensoría del Pueblo.

$4 \quad$ El Perú tiene una larga tradición en la producción minera, se lo ubica como primer productor mundial de plata; segundo de zinc y cobre (aunque The Economist de enero del 2011 lo señala como el tercer productor de cobre); tercero de estaño, bismuto, teluro y mercurio; cuarto de plomo y molibdeno; y quinto de oro.

$5 \quad$ El 19 de diciembre de 2012 el Pleno del Congreso aprobó la Ley 29968, "Ley de creación del Servicio Nacional de Certificación Ambiental para las Inversiones Sostenibles (SENACE)".

6 Información disponible en: http://www.cdi.org.pe/ 
Bajo este escenario de desaceleración económica, habría que tomar medidas para mantener las tasas de crecimiento. Debido a ello, el Poder Ejecutivo propone al Poder Legislativo un conjunto de medidas de reactivación económica (los denominados "paquetazos"), las mismas que se fueron dictando en diferentes momentos.

El 21 de noviembre de 2014 se propuso el Proyecto de Ley 3941 (en adelante, "Proyecto de Ley"), que contenía medidas ambientales como el fortalecimiento del SENACE, institución que recién se venía implementando ${ }^{7}$, a pesar de las expectativas generadas al momento de su creación respecto al fortalecimiento de la institucionalidad ambiental del país y, en específico, del Sistema Nacional de Evaluación de Impacto Ambiental (SEIA).

De acuerdo al Proyecto de Ley, una de las funciones que se le otorgaría al SENACE sería la de realizar el procedimiento de la Certificación Ambiental Global (CAG). La CAG busca reducir de manera gradual y progresiva los plazos de 450 días a 150 días de los procedimientos de aprobación de un EIA, sin perder la rigurosidad en las evaluaciones de cada uno de los permisos que contenga.

Finalmente, el Proyecto de Ley fue aprobado y el jueves 21 de mayo de 2015 se publicó la Ley 30327, "Ley de promoción de las inversiones para el crecimiento económico y desarrollo sostenible en las zonas de mayor exclusión social" (en adelante, "Ley 30327"). Recientemente, mediante Resolución Ministerial 290-2015-MINAM, se aprobó la propuesta de reglamento de dicha ley ${ }^{8}$. A pesar de que ya se ha cumplido el plazo establecido para su publicación, se espera que su implementación se traduzca en reales oportunidades para el necesario desarrollo ambiental.

En el presente artículo, se analizará la viabilidad de la CAG y también se identificarán los puntos críticos que el Estado debe fortalecer para que se cumplan los objetivos antes señalados.

\section{Desarrollo del Tema}

\section{A. Potenciales beneficios de la CAG}

$7 \quad$ El presupuesto asignado al SENACE fue de 12 millones de nuevos soles. Información disponible en:

http://www2.congreso.gob.pe/sicr/comisiones/2015/com2015precuegenrep.nsf//pubweb/03 5E1ACB0C1B6F3605257EDD000318A8/\$FILE/EXP_MINISTERIO_DE_AMBIENTE_PPTO_2016. PDF

Última fecha de consulta: 30 de octubre del 2016.

8 El viernes 30 de octubre de 2015 se publicó la Resolución Ministerial 290-2015-MINAM en la se dispone la publicación de la propuesta de Reglamento del Título II de la Ley 30327, sobre "Medidas para Optimizar y Fortalecer el Sistema Nacional de Evaluación de Impacto Ambiental". 
Antes de entender cuál es la función de la CAG, debemos tomar en cuenta que, en el Perú, los tiempos que le toma a un inversionista obtener cada uno de los permisos, licencias, derechos o autorizaciones vinculados, antes del inicio de operaciones de su proyecto, son muy extensos, pudiendo llegar a 5 años en el caso de proyectos mineros (en caso que no tenga mayores complicaciones).

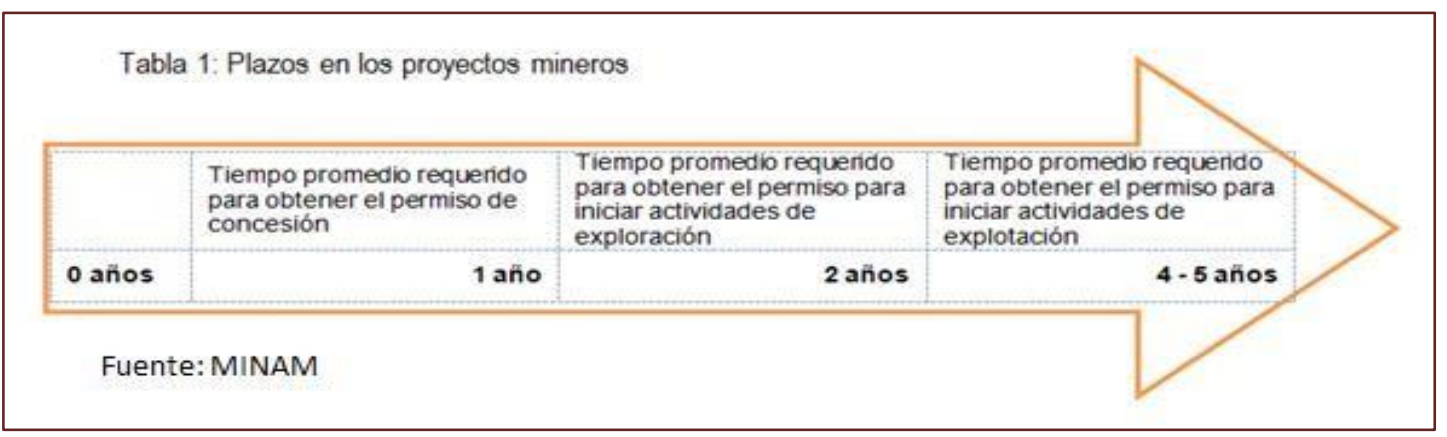

Así, para lograr una reducción de estos plazos, la CAG constituye un cambio estructural en el modo en cómo el Estado revisa y aprueba los EIA de proyectos de inversión de la categoría III, es decir, los EIA detallados (EIA-d). Al respecto, estos incluyen aquellos proyectos cuyas características, envergadura y/o localización, pueden producir impactos ambientales negativos significativos, cuantitativamente o cualitativamente, requiriendo un análisis profundo para revisar sus impactos y proponer la estrategia de manejo ambiental correspondiente ${ }^{9}$.

Por ello, en lugar de una revisión secuencial (un trámite tras otro), se realizará una revisión conjunta y en simultáneo por varias autoridades, tanto del EIA como de los permisos de naturaleza ambiental que estén vinculados. En consecuencia, las autoridades competentes que intervienen en la evaluación y viabilidad de los proyectos de inversión pública, pública privada, privada o de capital mixto deberán aprobar los títulos habilitantes señalados en la siguiente tabla:

$9 \quad$ Calle Valladares, Isabel, "Propuestas para el fortalecimiento del Sistema Nacional de Evaluación de Impacto Ambiental en el Perú". En: Sociedad Peruana de Derecho Ambiental, julio 2012. 


\begin{tabular}{|c|c|c|c|}
\hline \multicolumn{4}{|c|}{ Tabla 2: Procedimientos y Opiniones técnicas a integrar ${ }^{11}$} \\
\hline $\mathbf{N}^{\circ}$ & $\begin{array}{l}\text { AUTORIZACIÓN, LICENCIA, PERMISO, } \\
\text { OPINIÓN TÉNICA }\end{array}$ & ENTIDAD & PLAZO (Dias hábiles) \\
\hline 1 & $\begin{array}{l}\text { Aprobación de Estudios de Aprovechamiento } \\
\text { Hidrico. }\end{array}$ & \multirow{8}{*}{$\begin{array}{l}\text { Autoridad Nacional del } \\
\text { Agua - ANA }\end{array}$} & 45 \\
\hline 2 & $\begin{array}{l}\text { Autorización de Ejecución de Obras de } \\
\text { Aprovechamiento Hidrico. }\end{array}$ & & 45 \\
\hline 3 & $\begin{array}{c}\text { Autorización para ocupar, utilizar o desviar los } \\
\text { cauces, riberas, fajas marginales o los embalses } \\
\text { de las aguas. }\end{array}$ & & 45 \\
\hline 4 & $\begin{array}{c}\text { Autorización de Ejecución de Obras de defensa } \\
\text { ribereña y la utilización de materiales ubicados en } \\
\text { las fajas marginales. }\end{array}$ & & 45 \\
\hline 5 & $\begin{array}{c}\text { Autorización de uso de agua superficial o } \\
\text { subterránea. }\end{array}$ & & 45 \\
\hline 6 & $\begin{array}{l}\text { Autorización de Vertimientos de aguas Residuales } \\
\text { industriales, Municipales y Domésticas Tratadas. }\end{array}$ & & 45 \\
\hline 7 & $\begin{array}{c}\text { Autorización de Reúso de Agua Residuales } \\
\text { Tratadas. }\end{array}$ & & 45 \\
\hline 8 & $\begin{array}{l}\text { Opinión técnica vinculante para el otorgamiento de } \\
\text { autorizaciones de extracción de materiales de } \\
\text { acarreo en cauces naturales de agua. }\end{array}$ & & 45 \\
\hline 9 & $\begin{array}{l}\text { Autorización de desbosque a titulares de } \\
\text { operaciones y actividades distintas a la forestal. }\end{array}$ & $\begin{array}{l}\text { Servicio Nacional Forestal y } \\
\text { de Fauna Silvestre - } \\
\text { SERFOR }\end{array}$ & 45 \\
\hline 10 & Autorización Sanitaria para Tanque Séptico. & \multirow{3}{*}{$\begin{array}{l}\text { Dirección General de Salud } \\
\text { Ambiental - DIGESA }\end{array}$} & 45 \\
\hline 11 & $\begin{array}{c}\text { Opinión técnica favorable del sistema de } \\
\text { tratamiento y disposición sanitaria de aguas } \\
\text { residuales domésticas para } \\
\text { reúso. vertimiento y b) }\end{array}$ & & 45 \\
\hline 12 & $\begin{array}{l}\text { Opinión técnica favorable para el otorgamiento de } \\
\text { autorización de vertimiento y/o reúso de aguas } \\
\text { industriales tratadas: a) vertimiento, b) vertimiento }\end{array}$ & & 45 \\
\hline 13 & Derecho de Uso de Área Acuática. & $\begin{array}{c}\text { Dirección General de } \\
\text { Capitanias y Guardacostas } \\
\text { - DICAPI }\end{array}$ & 45 \\
\hline 14 & Estudio de Riesgo. & \multirow{2}{*}{$\begin{array}{l}\text { Organismo Supervisor de la } \\
\text { Inversión en Energia y } \\
\text { Mineria-OSINERGMIN }\end{array}$} & 45 \\
\hline 15 & Plan de Contingencia. & & 45 \\
\hline
\end{tabular}

Por ello, el objetivo es integrar estos procedimientos administrativos y opiniones técnicas en una Ventanilla Única de Certificación Ambiental (en adelante, "Ventanilla Única") a fin de brindar un servicio eficiente y eficaz que permita dar respuesta en los plazos legalmente establecidos, facilitando así el inicio de operaciones de un proyecto o actividad. De esta manera, se reducirá el tiempo que toma la tramitación secuencial de los procedimientos sin reducir los plazos de Ley.

Como se mencionó anteriormente, el procedimiento de evaluación debe permanecer a cargo del SENACE y entidades que otorgan su Opinión Técnica, el cual debe tener un plazo máximo de ciento cincuenta (150) días hábiles, con opción de ampliar hasta treinta (30) días hábiles máximo. Así pues, se dispone que todas las autoridades ambientales sectoriales remitan al SENACE los expedientes de los EIA aprobados, los cuales serán de disponibilidad pública en el Registro Administrativo de Certificaciones Ambientales. 
Es importante mencionar que la CAG no despoja a las entidades del Estado de sus competencias para otorgar los permisos de naturaleza ambiental y no reduce ni relaja de modo alguno las consideraciones técnico-ambientales para otorgarlos. Tanto la ANA, SERFOR, OSINERGMIN, DIGESA, entre otros, mantienen la competencia para otorgar sus permisos, pero con la diferencia de que lo realizarán en el marco del procedimiento de evaluación del Estudio Ambiental.

En consecuencia, podemos resumir los beneficios que se obtendrían de la participación del SENACE de la siguiente manera:

(i) reducción de tiempo para disponer de los títulos habilitantes que requerían la Certificación Ambiental,

(ii) títulos habilitantes evaluados de manera paralela y otorgados en un solo acto administrativo,

(iii) fomenta la ecoeficiencia, es decir, reduce el costo de papelería por aplicación de un proceso en línea a través de la Ventanilla Única,

(iv) seguimiento integrado a varios títulos habilitantes y la certificación ambiental, a través de un solo canal de comunicación con el Estado; y,

(v) disponibilidad de información consolidada y ordenada sobre los registros ambientales, incluyendo la línea base de los estudios ambientales aprobados

Para poder entender la parte procedimental de la CAG presentamos la información sistematizada por el MINAM en el Anexo 1, en donde se muestra gráficamente los beneficios en términos de reducción de tiempos.

\section{B. Situación internacional}

La Certificación Ambiental Global es una medida que ha sido implementada en otras partes del mundo. Ejemplos cercanos son la Licencia Ambiental ${ }^{10}$ otorgada por la Autoridad Nacional de Licencias Ambientales (ANLA) de Colombia y el Permiso Ambiental Sectorial (PAS) ${ }^{11}$ brindado por el Servicio de Evaluación Ambiental (SEA) en Chile. Tanto

10 Decreto 2820, Diario Oficial, n. 47.792, 5 de agosto de 2010, por el cual se reglamenta el título VII de la Ley 99 de 1993 sobre licencias ambientales.

11 Información disponible en: Sectoriales. 
la ANLA como el SEA son entidades de gobierno que concentran en un solo canal de comunicación los títulos habilitantes y la certificación ambiental ${ }^{12}$.

En el caso del Perú, la CAG busca agilizar el procedimiento dado los excesivos plazos que representa la gestión ambiental de un proyecto, en particular, de las actividades extractivas. En relación con ello, Milagros Verástegui, secretaria general del SENACE, comenta que ambos ejemplos fueron aplicados a consecuencia del mal funcionamiento que Colombia y Chile estaban teniendo respecto de los procedimientos ambientales, lo cual era una de las causas de las menores tasas de crecimiento de sus respectivas economías.

De acuerdo al Foro Económico Global, la burocracia gubernamental ya no es la principal razón que afecta la competitividad de Colombia, no dejando de prestarle la atención correspondiente $^{13}$. En ese orden de ideas, la simplificación de trámites ambientales ha ayudado tanto a Colombia como a Chile a adquirir mayor competitividad.

Esto se demuestra en los resultados en el Reporte Global de Competitividad (RGC) 20142015, pues la calificación obtenida por Colombia en el Índice Global de Competitividad (IGC) mejoró al pasar de 4,19 a 4,23. Con este resultado, Colombia se ubicó en el puesto 66 entre 144 economías, mientras que el año anterior el país había ocupado la posición 69 entre 148 países. De igual manera, Chile continúa siendo el país más competitivo de América Latina.

12 Información disponible en: http://semanaeconomica.com/article/economia/medioambiente/162235-senace-no-queremos-generar-mas-de-la-tramitologia-que-se-critica/.

Última fecha de consulta: 30 de octubre del 2016.

13 Información disponible en: http://app.eltiempo.com/economia/sectores/foro-economicomundial-colombia- avanzo-cinco-lugares-en-competitividad/16390713

última fecha de consulta: 30 de octubre del 2016. 


\begin{tabular}{|lc|}
\hline Latin America Top 10 & \\
\cline { 2 - 2 } The Global Competitiveness Index 2014-2015 & Global rank \\
\hline Chile & 33 \\
\hline Panama & 48 \\
Costa Rica & 51 \\
\hline Brazil & 57 \\
Mexico & 61 \\
Peru & 65 \\
Colombia & 66 \\
Guatemala & 78 \\
\hline Uruguay & 80 \\
\hline El Salvador & 84 \\
\hline Source: The Global Competitiveness Report 2014-2015 \\
Note: $2014-2015$ rank out of 144 economies
\end{tabular}

Por otro lado, Milagros Verástegui añade que el SENACE, desde la aprobación de la Ley 30327, ha estado realizando actividades junto con la ANLA y el SEA para armar alianzas estratégicas regionales a fin de consolidarse como un "eje del Pacífico".

Asimismo, nos menciona que uno de los objetivos del SENACE es ser un referente regional respecto al tema de la CAG. Además de los beneficios de reducción de plazos en los procedimientos, generará más confianza y legitimidad en los EIA. En palabras de Verástegui la "CAG será el valor agregado que tanto la sociedad y las empresas solicitan".

\section{Desafíos pendientes para la implementación adecuada de la CAG en el Perú}

Teniendo en cuenta el escenario actual de la CAG, a continuación expondremos cuáles son, a la fecha, los retos pendientes para su adecuada implementación ${ }^{14}$ :

\section{(i) Eje Normativo}

Para que la CAG pueda ser implementada, se necesita la aprobación del Reglamento de la misma. La Primera Disposición Complementaria Final de la Ley $30327^{15}$ menciona que el

14 Los ejes han sido tomados en cuenta de los "Temas Claves de la Ley 30327" del SENACE.

15 Disposiciones Complementarias Finales - Ley 30327:

PRIMERA. Reglamentación del Título II: Mediante decreto supremo emitido por el ministro del Ambiente y refrendado por los sectores correspondientes, se establecen las disposiciones 
plazo para emitir una disposición reglamentaria del Título II respecto a las "Medidas para optimizar y fortalecer el Sistema Nacional de Evaluación del Impacto Ambiental", en las cuales se incluye a la CAG, será de 60 días hábiles contados a partir de la entrada en vigencia de dicha Ley.

A pesar que el pasado jueves 18 de agosto de 2015 se cumplió la fecha para emitir la reglamentación de este tema, el viernes 30 de octubre de 2015 se aprobó la Resolución Ministerial 290-2015-MINAM, en la cual el Ministerio del Ambiente (MINAM) pone en consulta pública una propuesta de reglamento del Título II de la Ley 30327 (en adelante, la Prepublicación del Reglamento).

Teniendo en cuenta lo anterior, uno de los cuestionamientos a la tan esperada disposición reglamentaria era si existía voluntad política de emitirlo. Ello se debe a que, sin reglamento, los cambios esperados no pueden plasmarse en la realidad y, a su vez, al ser esta norma parte de las medidas de reactivación económica, tampoco se alcanzaría el objetivo de un mayor crecimiento económico.

Así pues, vemos que pese a haber existido una demora en la Prepublicación del Reglamento, este gobierno muestra su interés de afianzar los objetivos trazados respecto a la reducción de plazos en los procedimientos ambientales, la cual se traduce en la integración de la CAG. Asimismo, la voluntad política también se demuestra al haber designado al nuevo jefe del SENACE ${ }^{16}$, cargo que estaba encargado a Milagros Verástegui. Dicha designación es un buen paso para el fortalecimiento de la institucionalidad por parte del SENACE.

Lo ideal es que el presente gobierno habilite de manera adecuada al SENACE para evitar retrocesos que son comunes en países como el Perú que no son institucionalmente sólidos. En unos meses estaremos frente a una próxima gestión gubernamental, la cual deberá seguir fortaleciendo la institucionalidad del país y de este nuevo organismo.

Ante ello, se espera que el resultado de la consulta pública de la Prepublicación del Reglamento clarifique los siguientes temas: supervisión de la línea base, clasificación de los estudios ambientales, criterios para el uso de la información de la línea base compartida de los EIA aprobados y la nómina de profesionales. La reglamentación de estos aspectos contribuirá a recuperar la credibilidad en la gestión de los proyectos

reglamentarias del Título II de la presente Ley, en un plazo que no excederá de sesenta (60) días hábiles contados a partir de la entrada en vigencia de la presente Ley. designó a Patrick Wieland Fernandini como jefe del SENACE. Información disponible en: nuevo-jefe-del-senace-y-ponen-a-consulta-publica-importante-proyecto-de-normaambiental/ Última fecha de consulta: 30 de octubre de 2016. 
ambientales donde la CAG, al certificar la calidad de estos proyectos y reducir plazos, contribuirá no sólo a la competitividad del país sino también a la legitimidad del Estado peruano, que hoy más que nunca requiere la contribución de las industrias extractivas en el financiamiento de la diversificación productiva.

En ese sentido, el real desafío de implementar el Reglamento de la Ley 30327 es que se cumpla con todas las condiciones necesarias y suficientes, como contar con soporte informático, presupuesto y capital humano, para así alcanzar el propósito de dicha Ley, consistente en la simplificación e integración de permisos y procedimientos ambientales, es decir, reducir los plazos de 450 a 150 días.

\section{(ii) Coordinación Interinstitucional}

Para el éxito del proceso de implementación de la CAG es fundamental repensar y mejorar la coordinación interinstitucional en lo ambiental, aspecto que representa un desafío para la gestión pública peruana.

Por un lado, la conflictividad en los territorios donde operan las mega-inversiones es resultado de inadecuadas políticas públicas que no dotan de servicios básicos y donde muchas veces el alcance de los proyectos a aprobar está desarticulado de las acciones de los gobiernos locales y regionales. En ese sentido, los permisos ambientales en temas sensibles, como lo hídrico o lo forestal, requieren un adecuado conocimiento por parte del Estado del territorio donde se realizará la inversión.

Adicionalmente a ello, se observa diferencias en la gestión pública de los proyectos. La coordinación interinstitucional entre las entidades públicas de nivel nacional es relativamente fluida, no sucediendo ello con las entidades regionales y locales que no muestran las mismas capacidades y que influirán en las decisiones que permitan efectivizar los plazos previstos.

En las entrevistas sostenidas con especialistas en el tema, se resalta que el SENACE se está anticipando a generar las condiciones necesarias para que la coordinación interinstitucional opere adecuadamente. Por ello el SENACE, en conjunto con las autoridades que emiten los títulos habilitantes para la CAG, ha concretado convenios interinstitucionales y diferentes reuniones de trabajo periódicas.

Asimismo, el SENACE ha intercambiado opiniones con las entidades responsables de la gestión ambiental de Chile, Colombia, España e incluso Alemania a fin de incorporar las buenas prácticas de estos países en los permisos ambientales, así como la organización del Estado para que los procesos sean ágiles. Aun así, se debe seguir profundizando para tener un arreglo institucional acorde a los objetivos del SENACE y en especial de las capacidades de la CAG. 


\section{(iii) Soporte}

Para poder explicar el eje de soporte, debemos tomar en cuenta que existen tres situaciones que lo conforman: (i) tecnología para la operatividad de la CAG, (ii) disponibilidad de infraestructura y (iii) contratación de personal calificado.

Respecto al primer punto, debemos considerar que uno de los elementos para que la CAG opere de manera completa es a través de la implementación de la Ventanilla Única que ofrece la oportunidad de ser el único canal/plataforma de coordinación de estos procedimientos administrativos ambientales ${ }^{17}$. En ese sentido, es necesario que el SENACE tenga los recursos necesarios para poder contar con una plataforma virtual donde se centralice una sola base de datos, a través de la adquisición de hardware y software.

Lo óptimo de la operatividad de la plataforma virtual es que se implemente en todos los sectores gubernamentales que van a participar en el procedimiento de otorgamiento de permisos ambientales. Es decir, los gobiernos locales y regionales deben contar con las mismas condiciones tecnológicas para que los procedimientos puedan realizarse en los plazos contemplados. Lo anterior, sin perjuicio de que la información deba centralizarse en la Ventanilla Única del SENACE.

El segundo punto va de la mano con el primero, ya que, si bien es cierto que la tecnología es la que ayuda a que dicha Ventanilla Única se lleve a cabo, se necesita tener disponibilidad de infraestructura. Se tiene entendido que la CAG, al tener que registrar información de todos los sectores (minería, hidrocarburos, electricidad), concentrará información de las inversiones de todo el país, lo cual conlleva a que el SENACE deberá tener la proporcionalidad de ambientes para cada sector a efectos de registrar la información de manera ordenada, transparente y segura.

Respecto al tercer punto, este ha sido el más criticado desde la aprobación de la Ley 30327 y ello debido a que para poder implementar la CAG existe una disponibilidad limitada de profesionales necesarios para las funciones de las autorizaciones de la CAG. Dada la complejidad de aprobación de un EIA-d, se necesita un grupo de profesionales calificados y técnicos expertos en diferentes disciplinas.

En consecuencia, el SENACE debe contratar personal al cual se le capacite y brinde asistencia técnica para que puedan encargarse de los procedimientos de evaluación de impacto ambiental y así facilitar las tareas de la CAG, para lo cual se recomienda diseñar módulos/programas de capacitación continuos, programas de formación a nivel universitario, así como semilleros de futuros técnicos de la entidad. Asimismo, frente a la

17 Sociedad Peruana de Derecho Ambiental, Implementación del SENACE: temas clave de reflexión, agosto 2014. 
alta rotación de los funcionarios del Estado, se deben adoptar los incentivos adecuados que motiven la permanencia de los funcionarios capacitados de la entidad, tales como la implementación de una línea de carrera profesional, escala de remuneraciones que respondan a estándares del mercado, entre otros ${ }^{18}$.

Para lograr este fin de contar con un grupo capacitado, se debe fortalecer la gestión de recursos humanos, que motive a crear capacidades técnicas y de gestión a niveles de gobiernos regionales y locales, dado que son ellos quienes desarrollarán los procedimientos $\mathrm{y}$, a su vez, resolverán los obstáculos que se presenten en la implementación. El Estado deberá regular las competencias de manera específica para evitar: burocracia, corrupción, interpretación contraria de normas, duplicidad de costos de la administración, entre otros.

En este aspecto se espera que la oferta educativa universitaria deba responder a las coordinaciones interinstitucionales por dotar de una adecuada educación ambiental recogida en el Decreto Supremo 017-2012-ED que aprueba la Política Nacional de Educación Ambiental (PNEA), que tiene como una meta llegar al 2021 con 20\% de instituciones en educación superior universitaria y no universitaria con logro ambiental destacado, de esta manera, se espera contar con profesionales preparados para la gestión de proyectos ambientales y facilitar la gestión pública correspondiente.

\section{(iv) Presupuestal}

El presupuesto asignado al SENACE antes de la Ley 30327 era apenas de 12 millones de nuevos soles. El 2015 se le asignó 16.70 millones de nuevos soles y para el 2016 se asignó 14.80 millones de nuevos soles ${ }^{19}$. Claudia González, representante de la ANLA de Colombia, comentó en una entrevista con la Sociedad Peruana de Derecho Ambiental (SPDA) que el presupuesto que recibe el ANLA no solo es por parte del Estado, sino que también es complementado por cooperación internacional ${ }^{20}$. En ese sentido, el presupuesto del SENACE para fortalecer el inicio de la implementación de un instrumento de gestión ambiental como la CAG debe ser complementado con gestiones de diferentes organismos bilaterales y multilaterales, que permitan acceder a asistencia técnica para las

18 Sociedad Peruana de Derecho Ambiental, Implementación del SENACE: temas clave de reflexión, agosto 2014.

Información disponible en:

http://www2.congreso.gob.pe/sicr/comisiones/2015/com2015precuegenrep.nsf//pubweb/03 5E1ACB0C1B6F3605257EDD000318A8/\$FILE/EXP_MINISTERIO_DE_AMBIENTE_PPTO_2016. PDF

Última fecha de consulta: 30 de octubre del 2016.

20 Información disponible en: $h t t p: / / w w w . a c t u a l i d a d a m b i e n t a l . p e / ? p=32066$

Última fecha de consulta: 30 de octubre del 2016. 
áreas de mayor sensibilidad, que son la implementación de software y hardware para la obtención de un sistema adecuado de Ventanilla Única, y para la capacitación de los recursos humanos.

\section{(v) Difusión}

Hemos visto aspectos logísticos para la implementación de la CAG; sin embargo, un aspecto relevante en los servicios que proveen los Estados son los aspectos comunicacionales.

En lo comunicacional hay varios planos. Un primer punto es la manera como el funcionario comunica las etapas de la gestión de un proyecto a la ciudadanía y a las autoridades locales y regionales, debiendo implementar para ello estrategias comunicacionales y territoriales.

El SENACE es consciente de que tiene que implementar las estrategias antes mencionadas de manera preventiva, de tal manera que logre desarrollar una cultura ambiental y que el ambiente no represente obstáculos para las inversiones.

Para la sostenibilidad de las políticas ambientales, y dado que los proyectos se suelen ejecutar en los ámbitos locales y regionales donde las autoridades responden a ciclos políticos, SENACE ha optado por establecer una Red Universitaria de Evaluación y Certificación Ambiental (RUEDA), la cual tiene como fin contribuir en el proceso de fortalecimiento de las capacidades en materia de Certificación Ambiental a cargo del SENACE, difundir la normativa relacionada a los EIA, promover la responsabilidad social de los estudiantes, profesores y la comunidad universitaria en general y propiciar el interés de la comunidad universitaria en la incorporación de los temas vinculados a los EIA $^{21}$. Dicha iniciativa estará acompañada de materiales de soporte, capacitaciones y materiales de difusión (charlas, trípticos).

Sin embargo, estas son acciones que representan buenas intenciones que, para lograr la sostenibilidad de las políticas ambientales, requieren de persistencia para modificar conductas ambientales que a la fecha se caracterizan por el cuestionamiento a la forma como opera el Estado peruano.

\section{Conclusiones y recomendaciones}

El tema ambiental ha dejado de ser visto como un costo para las empresas y como un obstáculo para las inversiones por parte de los países. Ahora este tema es un factor que determina la competitividad tanto de las empresas y como de los países.

21 Información disponible en: http://www.senace.gob.pe/rueda/ Última fecha de consulta: 30 de octubre del 2016. 
La implementación de la Certificación Ambiental Global es una medida que busca fortalecer la institucionalidad ambiental y su aporte a la competitividad. Por ello, al representar una innovación en la gestión ambiental debe recuperar la legitimidad de los EIA y la confianza de la población.

Como se aprecia, la prepublicación del Reglamento de la Ley 30327 permitirá recoger la voz de opiniones especializadas pero también de quienes toman las decisiones de inversión. En ese sentido, se espera contar con un instrumento consensuado y a la vez práctico para los propósitos del país.

Enfatizamos en el desarrollo de capacidades en gestión ambiental dado que las principales inversiones privadas se caracterizan por magnitudes que desbordan la actual base institucional pública y que, aunada a un manejo inadecuado de expectativas de las poblaciones donde operan, determina el nivel de conflictividad señalado, donde además la Certificación Ambiental Global debe resolver las trabas observadas en la etapa de pre inversión de un proyecto, mas no así la gestión de la etapa de inversión y post inversión.

Finalmente, es pertinente que el Estado y las entidades que inciden en la formulación de las políticas públicas tomen en cuenta los ejes propuestos en la presente publicación para que la gestión ambiental esté dotada de capacidad y efectividad al implementar la Certificación Ambiental Global y que este esfuerzo no sea una buena intención más. 
Certificación global ambiental: ¿Buenas intenciones?

\section{舀FORSETI}

\section{A. ANEXOS}

ANEXO 1

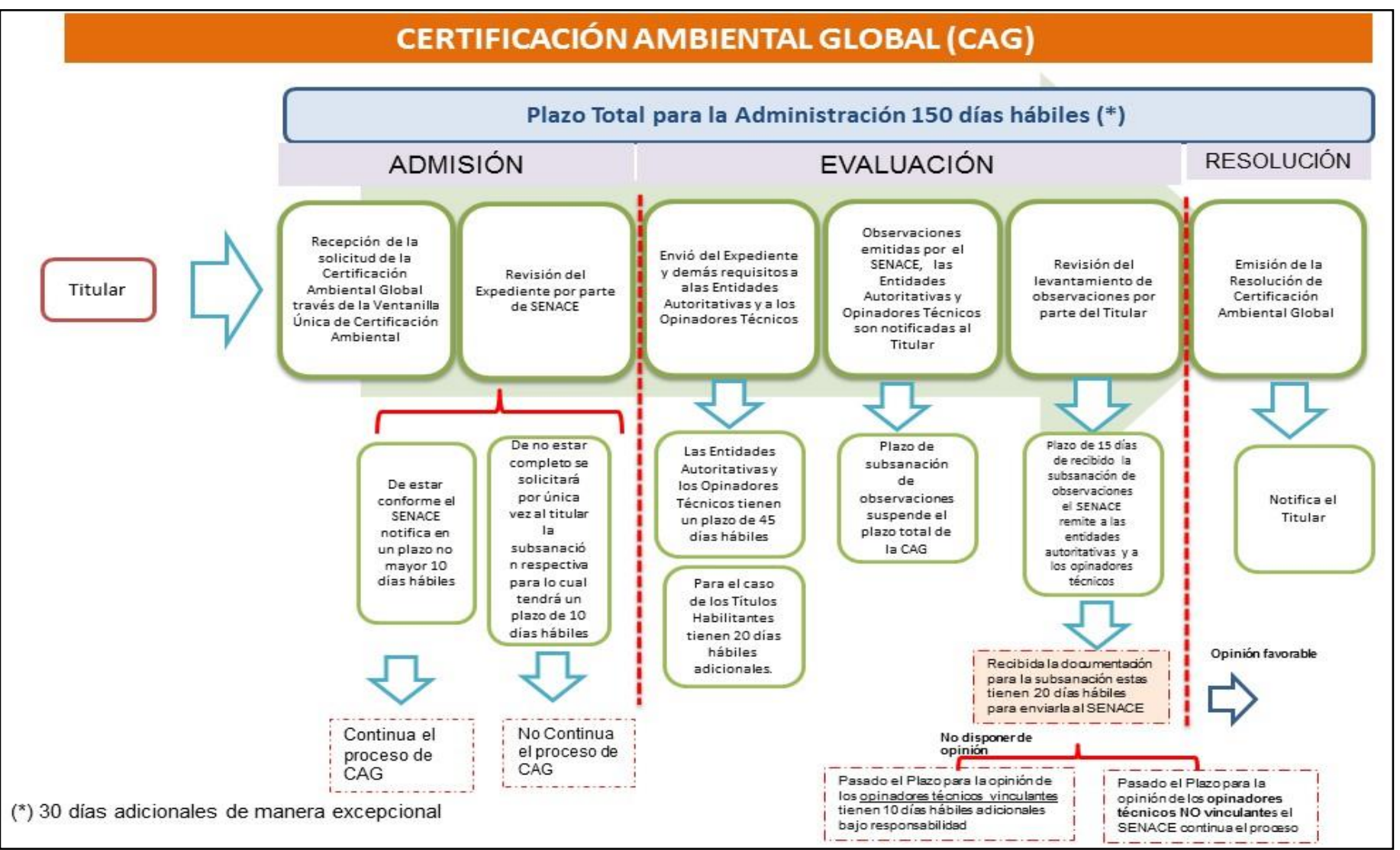

